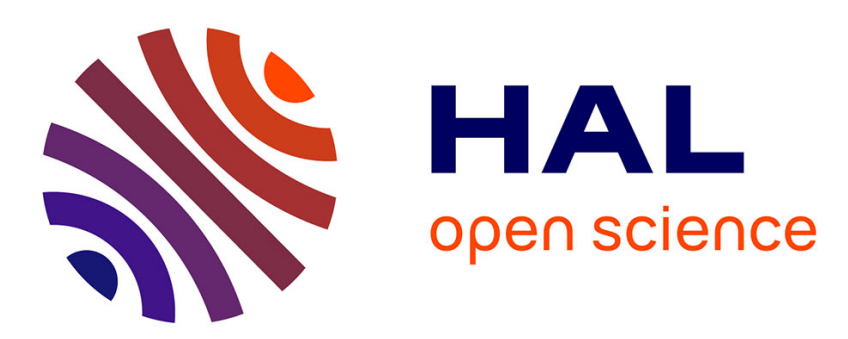

\title{
Modeling of particle cloud dispersion in compressible gas flows with shock waves
}

Guodong Gai, Olivier Thomine, Abdellah Hadjadj, Sergey Kudriakov

\section{To cite this version:}

Guodong Gai, Olivier Thomine, Abdellah Hadjadj, Sergey Kudriakov. Modeling of particle cloud dispersion in compressible gas flows with shock waves. Physics of Fluids, 2020, 32 (2), pp.023301. 10.1063/1.5135774 . hal-03105806

\section{HAL Id: hal-03105806 https://hal.science/hal-03105806}

Submitted on 11 Jan 2021

HAL is a multi-disciplinary open access archive for the deposit and dissemination of scientific research documents, whether they are published or not. The documents may come from teaching and research institutions in France or abroad, or from public or private research centers.
L'archive ouverte pluridisciplinaire HAL, est destinée au dépôt et à la diffusion de documents scientifiques de niveau recherche, publiés ou non, émanant des établissements d'enseignement et de recherche français ou étrangers, des laboratoires publics ou privés. 


\section{Modeling of particle cloud dispersion in compressible gas flows with shock waves}

Cite as: Phys. Fluids 32, 023301 (2020); https://doi.org/10.1063/1.5135774

Submitted: 08 November 2019 . Accepted: 14 January 2020 . Published Online: 03 February 2020

Guodong Gai (D), Olivier Thomine, Abdellah Hadjadj, and Sergey Kudriakov
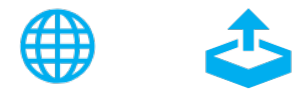

\section{ARTICLES YOU MAY BE INTERESTED IN}

Numerical study on shock-accelerated gas rings

Physics of Fluids 32, 026102 (2020); https://doi.org/10.1063/1.5135762

Stability of a vertical Couette flow in the presence of settling particles

Physics of Fluids 32, 024104 (2020); https://doi.org/10.1063/1.5140422

Linear and weakly nonlinear stability analysis on a rotating anisotropic ferrofluid layer

Physics of Fluids 32, 024101 (2020); https://doi.org/10.1063/1.5133102

\section{Sign up for topic alerts}

New articles delivered to your inbox 


\title{
Modeling of particle cloud dispersion in compressible gas flows with shock waves
}

\author{
Cite as: Phys. Fluids 32, 023301 (2020); doi: 10.1063/1.5135774 \\ Submitted: 8 November 2019 - Accepted: 14 January 2020 • \\ Published Online: 3 February 2020
}

Guodong Gai, ${ }^{1,2, a)}$ (D) Olivier Thomine, ${ }^{1}$ Abdellah Hadjadj, ${ }^{2}$ and Sergey Kudriakov

\author{
AFFILIATIONS \\ ${ }^{1}$ DEN-DM2S-STMF, Commissariat à l'Énergie Atomique et aux Énergies Alternatives, Université Paris-Saclay, \\ 91191 Gif-sur-Yvette, France \\ ${ }^{2}$ University of Normandy, INSA, CORIA UMR - 6614 CNRS, 76000 Rouen, France
}

a) Author to whom correspondence should be addressed: guodong.gai@cea.fr

\begin{abstract}
The effect of shock waves on the dispersion characteristics of a particle cloud is investigated both numerically and analytically. A onedimensional analytical model is developed for the estimation of the cloud topology in the wake of a shock wave, as a function of time, space, and characteristic response time $\tau_{p}$ of the cloud based on the one-way formalism. The model is compared with the results obtained with numerical simulations over a wide range of incident Mach numbers $M_{s}$ and particle volume fraction $\tau_{v, 0}$. An extension of the one-way formalism to the two-way is proposed by taking into account the post-shock gas deceleration due to the presence of particles. A significant increase in the cloud density is noticed. The effects of different parameters affecting the shock-spray interaction are elucidated and discussed. The two-way formalism is seen to better describe the effects of the particles on the propagation of the shock wave.
\end{abstract}

Published under license by AIP Publishing. https://doi.org/10.1063/1.5135774

\section{INTRODUCTION}

The interaction between shock waves and particles has been an active research field for decades. ${ }^{1-5}$ Many theoretical and experimental studies are conducted in order to understand the interaction mechanisms of shock waves with droplets or solid particles, ${ }^{6-10}$ since it is present and of major importance in various industrial applications. For instance, the compression waves can coalesce and generate shock waves in internal engines. ${ }^{11}$ The shocked fuel spray has different dispersion topologies, thus changing the combustion properties. Other applications concern explosion in the confinement building, where the shock waves can be initiated accidentally. In order to mitigate their effects, an aqueous foam ${ }^{12-15}$ or a water spray system ${ }^{16}$ can be used. In this case, the shock-spray interaction can change dramatically the dispersion of droplets, leading to the change in the mitigation capacity of the spray system. ${ }^{17-19}$ On the contrary, the particle cloud can also affect the propagation of the shock wave. $^{20}$

Basically, as a result of the high velocity of the shocked gas, the shock-droplet interaction can generate complex coupled phenomena such as droplet deformation, atomization, collision, coalescence, and evaporation. ${ }^{11,21,22}$ Moreover, the polydispersion of the droplets adds further difficulties to the investigations. To simplify the problem, various studies focus on the interaction between a shock wave and a single or an array of particles, ${ }^{23-25}$ where the effects of particles on gas flow are weak. Dense particle or particle curtains are also investigated, ${ }^{3,26}$ in which the collision between the particles is important.

Given the complexity of the droplet behavior during the interaction, rigid particles of uniform diameters are commonly used to simplify the shock-particle interaction. Even though the qualitative phenomena are well known, ${ }^{1,3}$ the interaction mechanisms between shock and particles are yet to be elucidated quantitatively in both well-conducted experiments and in numerical simulations and modelings. ${ }^{27}$ Particularly, the particle clouds of the volume fraction $\mathscr{O}\left(10^{-4}-10^{-3}\right)$ are of great interest in nuclear industrial applications.

The integral properties of the particle cloud movements such as volume fraction distribution and velocity distribution are also important for the large-scale simulations. ${ }^{28}$ However, to the best knowledge of the authors, the existing particle-resolved models for simulations of large-scale geometries such as nuclear confinement building are scarce, as a result of high computational expenses, especially for high Reynolds number flows. Thus, simple reduced-order 
modeling approaches and empirical correlations are considered to be the alternative solutions.

In this study, a new analytical model is developed to quantify the shocked gaseous flow impact on the dispersed phase using a oneway formalism. An extended two-way theoretical model is proposed, which takes into consideration the deceleration effect of the particles on the gas phase. The objectives of this study are threefold: (i) provide a simplified analytical formulation of particle cloud dispersion after the interaction with a shock wave, (ii) elucidate the importance of the two-way formalism on the description of the shock-cloud interaction, and (iii) identify the main parameters and their effects on the shock-cloud interaction. The theoretical model is validated with high-resolved numerical simulations.

This paper is organized as follows: Section II discusses the characteristics of the particle cloud. Section III presents an analytical formulation of particle dispersion with a shock wave. Section IV discusses the assessment of the analytical model, and the comparisons between the analytical results and the numerical simulations are presented in Sec. V. Finally, the main conclusions together with recommendations for future work are given in Sec. VI.

\section{CHARACTERISTICS OF THE CLOUD PARTICLES}

In this study, assumptions are made so that the gas is considered as inviscid and follows the perfect-gas law, the particles are supposed to be rigid and spherical, with small volume fractions, the collisions between them are neglected, ${ }^{29}$ only viscous drag forces act on the particles, and the heat transfer between gas and particles is neglected.

Initially at rest, the particles are assumed to be uniformly distributed throughout the computational domain. After the passage of the shock, the particles are accelerated by the gas flow. In order to determine the evolution of the particles, we compute the force applied by the flow of velocity $\mathbf{u}(\mathbf{x}, t)$ on a spherical particle of coordinate $\mathbf{x}$, with a velocity $\mathbf{V}(t)$ and a diameter $d_{p}$. The general equation of motion reads

$$
m_{p} \frac{d \mathbf{V}(\mathbf{t})}{d t}=\sum \mathbf{F},
$$

where $m_{p}=\pi \rho_{p} d_{p}^{3} / 6$ is the particle mass and $\rho_{p}$ is the particle density. Here, we neglect the gravity, the Magnus' force, and the Basset force as a result of the high ratio between the densities of the liquid and gas phases. The viscous drag force gives

$$
\mathbf{F}=\frac{\pi}{8} \rho_{p} d_{p}^{2} C_{D}|\mathbf{u}(\mathbf{x}, t)-\mathbf{V}(\mathbf{t})|(\mathbf{u}(\mathbf{x}, t)-\mathbf{V}(\mathbf{t})),
$$

where $C_{D}$ is the drag coefficient of the particles defined as

$$
C_{D}=\frac{24}{R e_{p}}, \quad \text { with } R e_{p}=\frac{\rho_{g}|\mathbf{u}(\mathbf{x}, t)-\mathbf{V}(\mathbf{t})| d_{p}}{\mu_{g}},
$$

where $R e_{p}$ is the particular Reynolds number related to the flow around the particle and $\mu_{g}$ is the dynamic viscosity of the gas. The diameters of the particles considered in this study vary from $10 \mathrm{~nm}$ to $50 \mu \mathrm{m}$. Due to the small size of particles, the drag coefficient is given by the Stokes coefficient for laminar flow. The equation of motion for each particle can be obtained as

$$
\frac{d \mathbf{V}(\mathbf{t})}{d t}=\frac{1}{\tau_{p}}(\mathbf{u}(\mathbf{x}, t)-\mathbf{V}(\mathbf{t})) \text {, with } \tau_{p}=\frac{\rho_{p} d_{p}^{2}}{18 \mu_{g}}
$$

In the case of a two-way interaction, and in order to estimate the effect of the particles on the gas, the momentum conservation is taken into consideration. For a gas volume $\mathscr{V}$ containing one particle with a velocity variation $\frac{d \mathbf{V}}{d t}$, the particle can decelerate the gas with respect to the following equality:

$$
\frac{d \mathbf{u}}{d t}=-\frac{m_{p}}{\rho_{g} \mathscr{V}} \frac{d \mathbf{V}(\mathbf{t})}{d t} .
$$

\section{ANALYTICAL DETERMINATION OF PARTICLE DISPERSION WITH SHOCK WAVE}

\section{A. Eulerian cloud velocity}

In the one-way formalism, the evolution of the particles allows us to determine analytically their velocities and coordinates as a function of time, when a constant velocity gas is applied. Let $M_{s}$ denote the Mach number of the shock wave. The pre- and the postshock gas properties can be found in Appendix A. Consider any point in the particle-laden domain at a time $t$, with the position $x$, denoted as $(x, t)$. The time origin corresponds to the beginning of the cloud interaction, with the shock initially at $x=0$. For each point in the $(x, t)$ diagram (see Fig. 1), two configurations are possible, depending on whether the shock wave has already passed the interface $\left(x \geq M_{s} c t\right)$ or not ( $c$ being the sound speed in the gas at rest).

It is possible to calculate the initial position and time of each particle. Let $x^{\prime}$ be the distance covered by the particle after the interaction with the shock, and $t^{\prime}$ the duration of the interaction. The distance covered by the particle during $t^{\prime}$ is $x^{\prime}=x\left(t^{\prime}\right)=x-x_{0}$, and the distance covered by the shock wave is $M_{s} c t^{\prime}=M_{s} c t-x_{0}$. Knowing the shock velocity, $V_{s}=M_{s} c$, and the gas velocity behind the shock $u_{g}$, one can deduce from Eq. (4) the velocity as well as the distance covered by a particle $x^{\prime}$ as a function of time $t^{\prime}$ during which it is exposed to the gas of a velocity $u_{g}$,

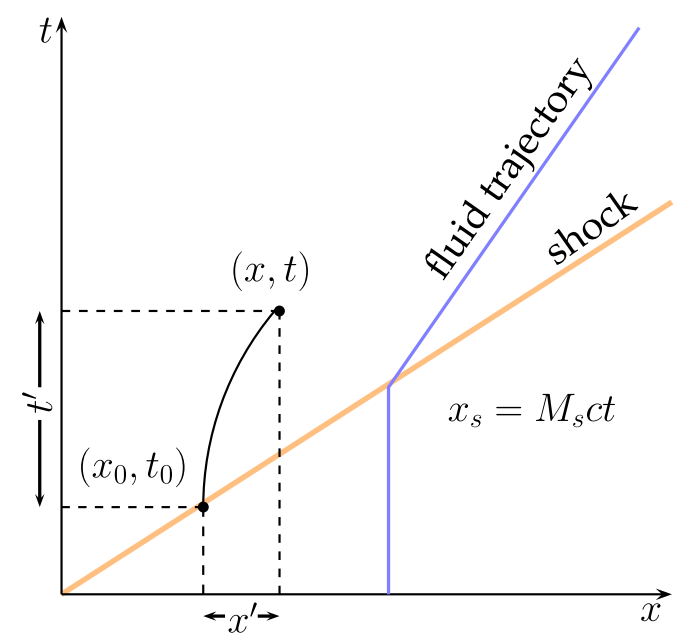

FIG. 1. Space-time diagram $(x, t)$ of the considered system with $x_{S}$ the shock position, $x^{\prime}$ the distance covered by a particle located initially at $x_{0}$, and $t^{\prime}$ the duration of the interaction of the particle with the shock. 


$$
\left\{\begin{array}{c}
V\left(t^{\prime}, \tau_{p}, u_{g}\right)=u_{g}\left(1-e^{-t^{\prime} / \tau_{p}}\right) \\
x\left(t^{\prime}, \tau_{p}, u_{g}\right)=\int_{0}^{t^{\prime}} V\left(t, \tau_{p}, u_{g}\right) d t=u_{g}\left(t^{\prime}-\tau_{p}\left(1-e^{-t^{\prime} / \tau_{p}}\right)\right) .
\end{array}\right.
$$

The two unknown variables $x^{\prime}$ and $t^{\prime}$ satisfy the following relations:

$$
\left\{\begin{array}{c}
x^{\prime}=x\left(t^{\prime}, \tau_{p}, u_{g}\right)=x-x_{0} \\
M_{s} c t^{\prime}=M_{s} c t-x_{0} .
\end{array}\right.
$$

By excluding $x_{0}$ from Eq. (7) and substituting the expression for $x^{\prime}$ from Eq. (6), one can deduce that

$$
u_{g} \tau_{p}\left(e^{-t^{\prime} / \tau_{p}}-1\right)+M_{s} c t-x=\left(M_{s} c-u_{g}\right) t^{\prime} .
$$

Solving this equation (cf. Appendix B) gives the following expression:

$$
t^{\prime}\left(x, t, u_{g}\right)=\tau_{p} \mathscr{W}\left(\frac{u_{g} e^{\eta}}{M_{s} c-u_{g}}\right)+\frac{M_{s} c t-u_{g} \tau_{p}-x}{M_{s} c-u_{g}},
$$

where

$$
\eta=\frac{u_{g} \tau_{p}-M_{s} c t+x}{\tau_{p}\left(M_{s} c-u_{g}\right)} .
$$

The Lambert function $\mathscr{W}^{30}$ is defined implicitly as the solution of the equation $\alpha \exp (\alpha)=\beta$ (see Appendix B). It is also possible to obtain the Eulerian velocity of the cloud $u$ as

$$
u\left(x, t, \tau_{p}, u_{g}\right)=V\left(t^{\prime}\left(x, t, u_{g}\right), \tau_{p}, u_{g}\right)=u_{g}\left(1-\exp \left(-t^{\prime}(x, t) / \tau_{p}\right)\right) .
$$

\section{B. Mean cloud density}

Using the conservation of mass, it is possible to determine the global spray characteristics in the post-shock area. If one considers that the time $t$ of the interaction of the shock with the cloud is very large with respect to the response time $\tau_{p}$, the first particle distance covered can be approximated with $x\left(t, \tau_{p} / t \rightarrow 0\right)=u_{g} t$. It allows us to determine the cloud length in the post-shock area as

$$
L\left(t, \tau_{p} / t \rightarrow 0, u_{g}\right)=M_{s} c t-x\left(t, \tau_{p} / t \rightarrow 0, u_{g}\right)=\left(M_{s} c-u_{g}\right) t .
$$

Considering that the particles are solid and undeformable, the initial cloud length is $M_{s} c t$ and becomes $\left(M_{s} c-u_{g}\right) t$, we deduce that the post-shock density of the particles $\overline{\tau_{v}}$ can be linked to the pre-shock density $\tau_{v, 0}$ by

$$
\overline{\tau_{v}}\left(t, \tau_{p}, u_{g}\right)=\tau_{v, 0} \frac{M_{s} c}{M_{s} c-u_{g}} .
$$

The initial and the post-shock cloud lengths are represented in Fig. 2(b). Using Eq. (A1), one can obtain

$$
\frac{\overline{\tau_{v}}}{\tau_{v, 0}}=\frac{1}{\frac{\gamma-1}{\gamma+1}+\frac{2}{\gamma+1} \frac{1}{M_{s}^{2}}} .
$$

The evolution of the ratio $\overline{\tau_{v}} / \tau_{v, 0}$ as a function of $M_{s}$ is given in Fig. 2(a). One can see that when $M_{s} \rightarrow \infty, \overline{\tau_{v}} / \tau_{v, 0}$ approaches $(\gamma+1) /(\gamma-1)=6.0$ for air, a value that $\overline{\tau_{v}} / \tau_{v, 0}$ can never exceed.

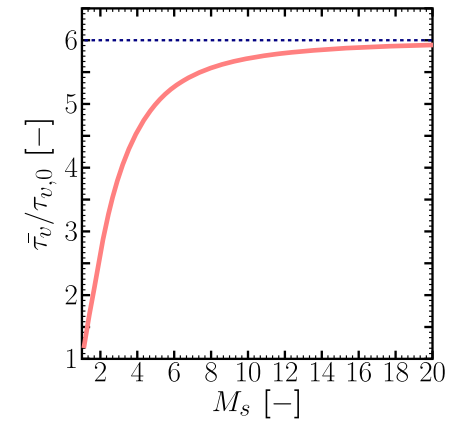

(a)

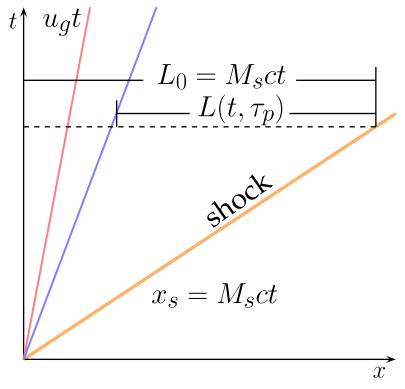

(b)
FIG. 2. Cloud density as a function of the Mach number and the length of cloud in the post-shock area. (a) Evolution of $\overline{\tau_{v}} / \tau_{v, 0}$ as a function of $M_{s}$; the dashed blue line represents the asymptotic limit given by $(\gamma+1) /(\gamma-1)$ with $\gamma=1.4$. (b) Space-time diagram showing the cloud length pre- and post-shock.

When the inertia of the particles cannot be neglected, one can obtain a mean load rate after the shock passage using Eqs. (6) and (12),

$$
\overline{\tau_{v}}\left(t, \tau_{p}, u_{g}\right)=\tau_{v, 0} \frac{M_{s} c}{M_{s} c-u_{g}+u_{g} \frac{\tau_{p}}{t}\left(1-\exp \left(-t / \tau_{p}\right)\right)} .
$$

Equations (13) and (15) show accumulation of particles after the shock at the contact surface. The evolutions of the particle load rate for different $\tau_{p}$ and for a fixed $M_{s}=1.1$ are given in Fig. 3. We can see that the time necessary to reach the asymptotic value for the particle load rate increases with particle response time as expected.

\section{Eulerian cloud density evolution}

One more hypothesis is necessary to estimate the Eulerian cloud density evolution. Let us assume that the particles are initially regularly disposed with a mean distance of $\Delta x_{0}$ between them. The

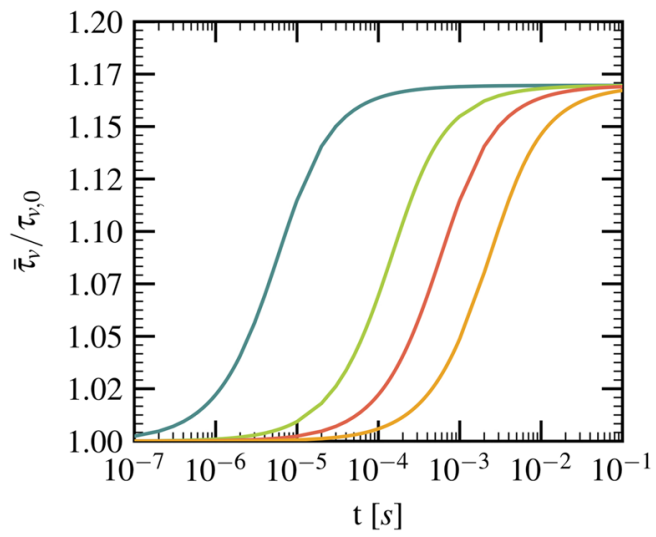

FIG. 3. Mean load rate evolutions for particles of different $\tau_{p}$ in air: $\tau_{p}=3 \times 10^{-6} \mathrm{~s}$ (blue solid line), $\tau_{p}=7.5 \times 10^{-5} \mathrm{~s}$ (green solid line), $\tau_{p}=3 \times 10^{-4} \mathrm{~s}$ (red solid line), $\tau_{p}=1.2 \times 10^{-3} \mathrm{~s}$ (orange solid line), $\rho_{p}=10^{3} \mathrm{~kg} / \mathrm{m}^{3}, M_{s}=1.1$, and $u_{g}=55.19 \mathrm{~m} / \mathrm{s}$ 


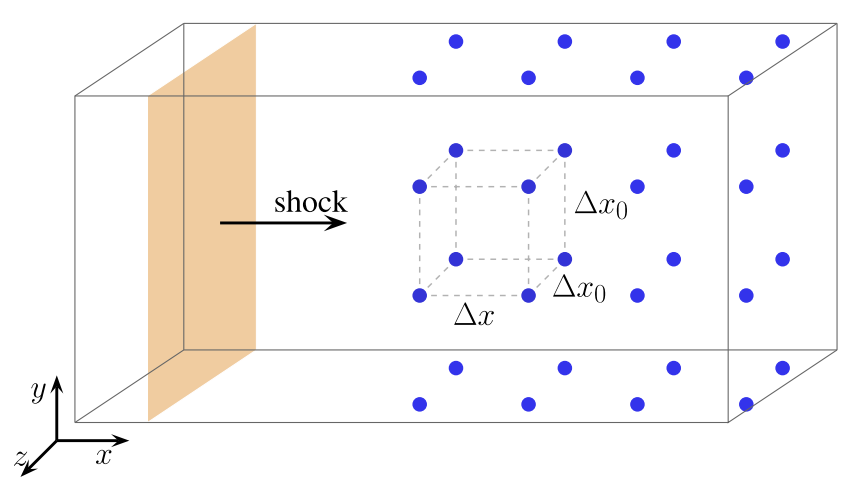

FIG. 4. Initial configuration of the shock/particle interaction.

cloud is also initially structured according to a cubic particle shape. The load rate $\tau_{v, 0}$ of this cloud is also the ratio between the volume taken by the particles and the volume of the gas. The initial organization of the particles and the initial load rate are shown in Fig. 4. With such a cubic arrangement, the load rate is

$$
\tau_{v, 0}=\frac{\pi d_{p}^{3}}{6 \Delta x_{0}^{3}} .
$$

Knowing that the shock propagates along the $x$-direction, the load rate of the shocked particle-laden region can be

$$
\tau_{v}\left(t, \tau_{p}, u_{g}\right)=\frac{\pi d_{p}^{3}}{6 \Delta x_{0}^{2} \Delta x\left(t, \tau_{p}, u_{g}\right)} .
$$

Let us consider two neighbor particles on the $x$-axis at initial positions $x_{2}^{0}$ and $x_{1}^{0}$ such as $x_{2}^{0}-x_{1}^{0}=\Delta x_{0}$. With previous results, it is possible to estimate the time $t^{\prime}$ during which the particles are in the post-shock area. Let us denote $\Delta t=\Delta x_{0} / M_{s} c$, the interval time taken by the shock to cover the inter-particle distance. The distance between these two particles as a function of $t^{\prime}$ is

$$
\begin{aligned}
\Delta x\left(t^{\prime}, \tau_{p}, u_{g}\right) & =x_{2}\left(t^{\prime}-\Delta t, \tau_{p}, u_{g}\right)-x_{1}\left(t^{\prime}, \tau_{p}, u_{g}\right) \\
& =\left(x_{2}^{0}+x\left(t^{\prime}-\Delta t, \tau_{p}, u_{g}\right)\right)-\left(x_{1}^{0}+x\left(t^{\prime}, \tau_{p}, u_{g}\right)\right) \\
& =\Delta x_{0}+x\left(t^{\prime}-\Delta t, \tau_{p}, u_{g}\right)-x\left(t^{\prime}, \tau_{p}, u_{g}\right)
\end{aligned}
$$

Substituting Eqs. (6) and (16) into (18) and according to the definition of $\Delta t$ and $\tau_{p}$, one can deduce

$$
\begin{aligned}
\Delta x= & \Delta x_{0}\left(1-\frac{u_{g}}{M_{s} c}\right)+u_{g} \tau_{p} \exp \left(-t^{\prime} / \tau_{p}\right) \\
& \times\left[\exp \left(\sqrt{\frac{18 \mu_{g} \tau_{p}}{\rho_{p}}} \sqrt[3]{\frac{\pi}{6 \tau_{v, 0}}} \frac{1}{M_{s} c \tau_{p}}\right)-1\right] .
\end{aligned}
$$

Dividing Eq. (19) by $x_{0}$, one can obtain according to Eqs. (16), (17), and the definition of $\tau_{p}$,

$$
\frac{\tau_{v}\left(t^{\prime}, \tau_{p}, u_{g}\right)}{\tau_{v, 0}}=[\left(1-\frac{u_{g}}{M_{s} c}\right)+\underbrace{\sqrt{\frac{\rho_{p}}{18 \mu \tau_{p}}} \sqrt[3]{\frac{6 \tau_{v, 0}}{\pi}} u_{g} \tau_{p} \exp \left(-t^{\prime} / \tau_{p}\right)\left(\exp \left(\sqrt{\frac{18 \mu_{g} \tau_{p}}{\rho_{p}}} \sqrt[3]{\frac{\pi}{6 \tau_{v, 0}}} \frac{1}{M_{s} c \tau_{p}}\right)-1\right)}_{\mathscr{A}}]^{-1} .
$$

Two evident conclusions can be deduced. The term $\mathscr{A}$ is always positive and

$$
\tau_{v}\left(t^{\prime}, \tau_{p}, u_{g}\right)<\tau_{v, 0} \frac{M_{s} c}{M_{s} c-u_{g}}=\tau_{v, \max }
$$

which is the maximal value of the post-shock density estimated by Eq. (15). In the one-way formalism, we can conclude that for any physical parameters, the maximal density of the cloud can never exceed the mean density that a null-inertia cloud could have. The second point is that, for a very low $\tau_{p}$, one can have $\mathscr{A} \rightarrow 0$. In this case, the cloud density increases to a constant value $\tau_{v, \max }$. This model is applicable for various particle cloud density ratios $\rho_{p} / \rho_{g}$, provided that the gravity of the particles is negligible compared to the drag force.

\section{Extension to two-way formalism}

With the existence of the particle-gas interaction, the gas velocity decreases due to the conservation of momentum. We can assume that this velocity is reduced by a value $\varepsilon\left(u_{g}\right)$. The next particle will relax to a velocity of $u_{g}-\varepsilon\left(u_{g}\right)$. Figure 5 shows the acceleration of two successive particles by the shock wave in the two-way model. In this case, the load rate will severely increase at the cloud extremity. In addition, the presence of particles can slow down the postshock gas velocity. It is at the cloud extremity that the particles slipping velocity is the highest. It is also at this location that the gas is most impacted by the presence of particles, and that $\varepsilon$ has the

(a)
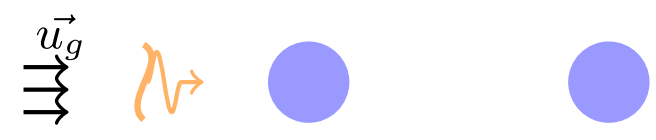

(b)
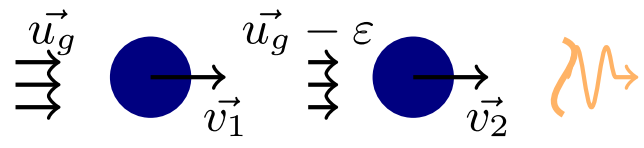

FIG. 5. Sketch of two successive particle motion (a) before and (b) after the shock passage. 
highest values. Knowing that the density and the local velocity of the cloud have no analytical solutions, one can only determine the mean characteristics of the cloud after the shock.

First, it is assumed that the Mach number of the transmitted shock takes a constant value equal to $M_{s}=1.1$ in the two-way modeling. According to numerical simulations, this assumption is justified for small particle volume fractions $\tau_{v, 0}<10^{-3}$. Our attempt is to obtain the mean post-shock gas velocity $\tilde{u}_{g}$. Let us consider a volume element $\mathscr{V}$ inside, which is the particle volume $\tau_{v, 0} \mathscr{V}$. This analysis is considered in the case where the particles have completely relaxed to the post-shock gas velocity $\tilde{u}_{g}$. With respect to the kinetic energy conservation, one can directly deduce

$$
\rho_{g} u_{g}^{2} \mathscr{V}=\rho_{g}^{\prime} \tilde{u}_{g}^{2} \mathscr{V}^{\prime}+\tau_{v, 0} \mathscr{V}^{\prime} \rho_{p} \tilde{u}_{g}^{2}, \text { where } \mathscr{V}^{\prime} \simeq \mathscr{V}\left(1-\frac{u_{g}}{M_{s} c}\right)
$$

and

$$
\frac{\rho_{g}^{\prime}}{\rho_{g}}=\frac{(\gamma+1) M_{s}^{2}}{2+(\gamma-1) M_{s}^{2}},
$$

where $\rho_{p}$ is the density of the particles. So, we obtain

$$
\tilde{u}_{g}=\frac{u_{g}}{\sqrt{1-\frac{u_{g}}{M_{s} c}} \sqrt{\frac{(\gamma+1) M_{s}^{2}}{2+(\gamma-1) M_{s}^{2}}+\tau_{v, 0} \frac{\rho_{p}}{\rho_{g}}}} .
$$

Taking the mean load rate given by Eq. (15), we have

$$
\tilde{\tau}_{v}=\tau_{v, 0} \frac{M_{s} c}{M_{s} c-\tilde{u}_{g}}
$$

\section{ASSESSMENT OF THE ANALYTICAL MODEL}

Here, we consider the numerical simulation of the interaction between a shock wave and a gas-particle in the two-phase mixture as illustrated in Fig. 6. This is a basic configuration commonly used to study shock wave attenuation particle-laden regions. ${ }^{31}$ A piston moving at a speed $u_{g}$ can generate a shock traveling at a velocity $V_{s}$ (see Appendix A).

contact surface

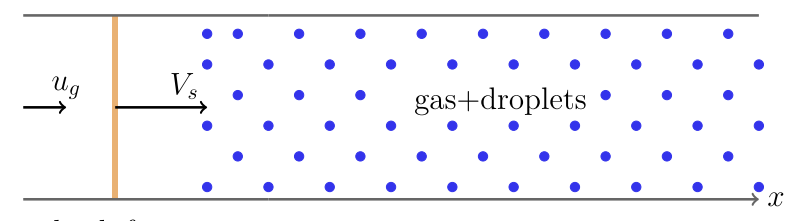

shock front

contact surface

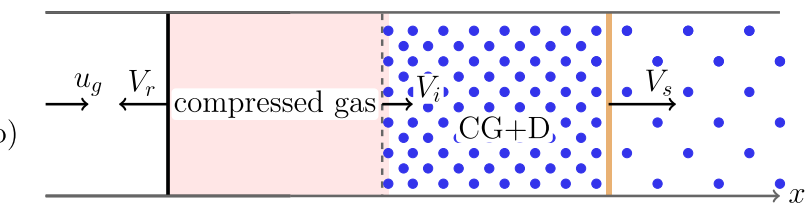

reflected pressure wave

transmitted shock front

FIG. 6. Sketch of the shock and contact surface before (a) and after the interaction (b); CG: compressed gas, D: droplets.
TABLE I. Post- and pre-shock gas flow characteristics, $M_{s}=1.1, \rho_{p} / \rho_{g}=553.7$.

\begin{tabular}{lcc}
\hline \hline Gas flow parameters & Post-shock & Pre-shock \\
\hline$u_{g}(\mathrm{~m} / \mathrm{s})$ & 58.21 & 0 \\
$\rho_{g}\left(\mathrm{~kg} / \mathrm{m}^{3}\right)$ & 1.21 & 1.04 \\
$p_{g}(\mathrm{bar})$ & 1.25 & 1.01 \\
$T_{g}(\mathrm{~K})$ & 396 & 370 \\
\hline \hline
\end{tabular}

The simulations are conducted using an in-home compressible Navier-Stokes code named Asphodele, developed in CORIA laboratory Rouen France. ${ }^{32}$ The Eulerian-Lagrangian approach is used with an Unresolved Discrete Particle Model (UDPM). The space discretization uses a fifth-order WENO (weighted essentially non-oscillatory) scheme with global Lax-Friedrichs splitting. ${ }^{33} \mathrm{~A}$ third-order Runge-Kutta method is adopted for time marching. The minimal storage time-advancement scheme ${ }^{34}$ is used to reconstruct the Runge-Kutta method for the temporal resolution. The onedimensional computational domain $L_{0}=1 \mathrm{~m}$ consists of 1000 points, with 1000 particles initially defined in each elementary cell.

The analytical model and the numerical results are compared together in this section. The difference between theoretical and numerical cloud velocities in the one-way formalism is first studied. As illustrated in Fig. 6, the shock wave and the contact surface are initially located at $x_{0}=0$. These characteristics of the gas in the preand the post-shock domain are given in Table I.

The cloud velocity and the gas velocity are studied for particles with five distinct diameters ranging from nano to micro meters. The particles have a mass density of $\rho_{p}=664.4 \mathrm{~kg} / \mathrm{m}^{3}$ at atmospheric temperature and pressure corresponding to a given gas (here, we consider cycloheptene $\mathrm{C}_{7} \mathrm{H}_{16}$, as an example). Table II gives the particle diameters and the related equivalent characteristic response time $\tau_{p}$. In what follows, we choose the initial pre-shock properties as characteristic scales such as $u_{g, 0}, \tau_{v, 0}$, and $P_{0}$. The length of the calculation domain $L_{0}$ is chosen as the characteristic scale of the coordinates.

For very small particles $\left(d_{p}=10 \mathrm{~nm}\right.$ and $\left.d_{p}=1 \mu \mathrm{m}\right)$, one can assume that their velocity increases rapidly toward the gas velocity and coincides with it. As a consequence, two areas are noted in Figs. 7(a) and 7(b): the pre-shock area, where both particles and gas are at rest, and the post-shock area, where the gas and the particles velocity are equal to $u_{g}$.

In the case where the particles inertia cannot be neglected, they progressively accelerate to relax toward the gas velocity.

TABLE II. Diameter of particles and corresponding equivalent characteristic response time $\tau_{p}$.

\begin{tabular}{cc}
\hline \hline Droplet diameter $d_{p}(\mu \mathrm{m})$ & Response time $\tau_{p}(\mu \mathrm{s})$ \\
\hline 0.01 & $1.57510^{-4}$ \\
1 & 1.575 \\
10 & 157.5 \\
20 & 630 \\
50 & 3937 \\
\hline
\end{tabular}




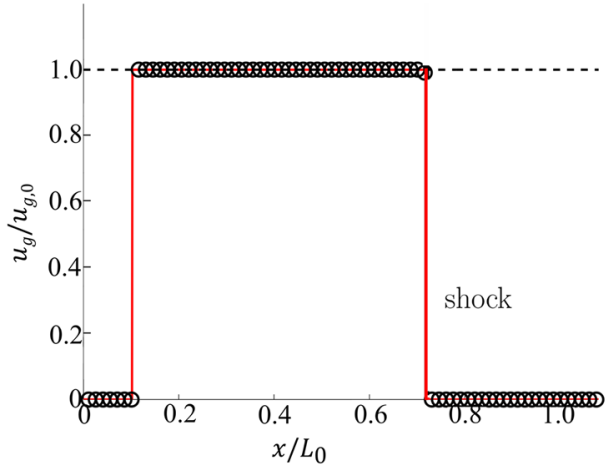

(a)

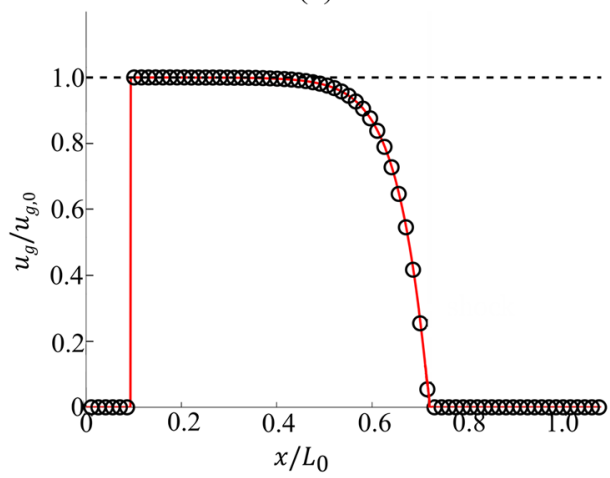

(c)

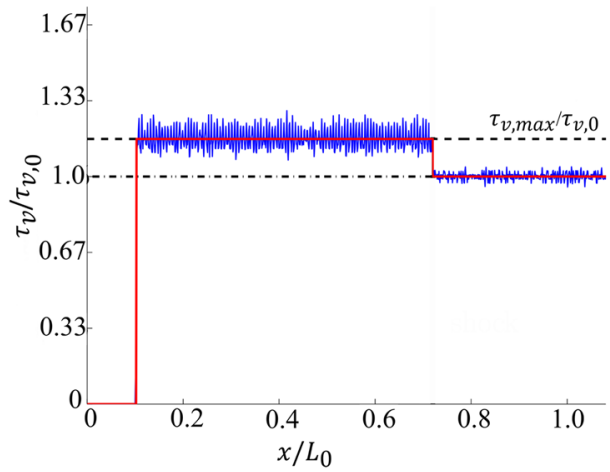

(a)

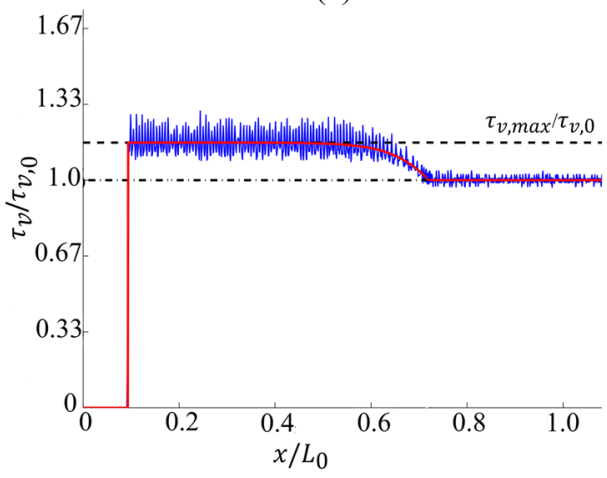

(c)

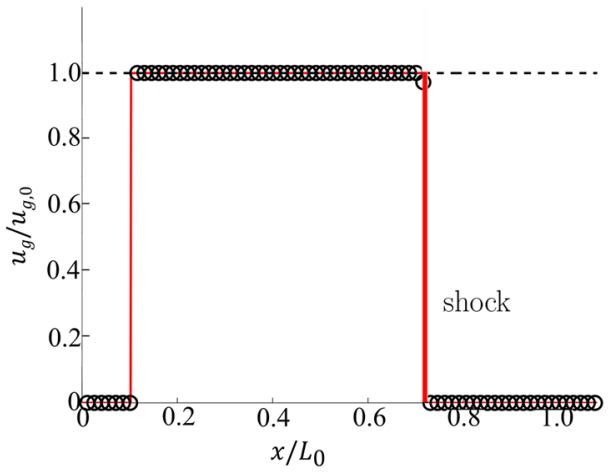

(b)

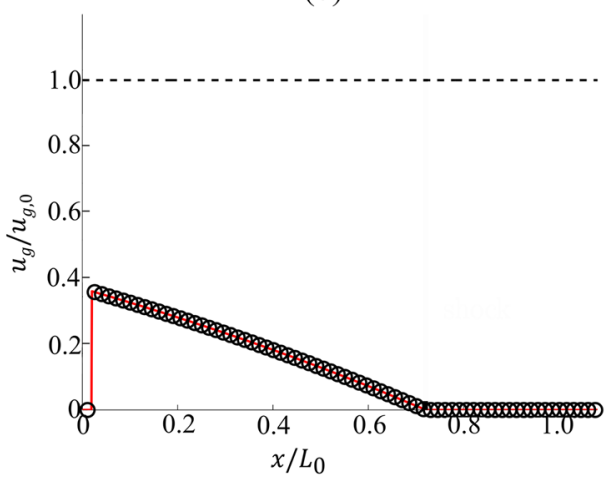

(d)

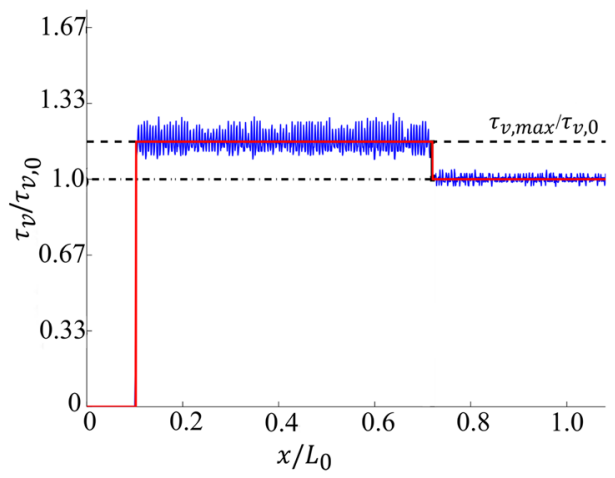

(b)

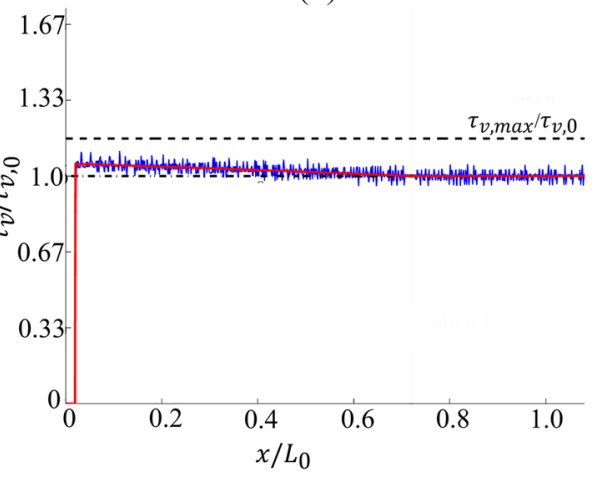

(d)
FIG. 7. Eulerian cloud velocity within the one-way formalism. Numerical results (black circle), theoretical model (red solid line), and their maximal value $\left(u_{g}\right)$ for different diameters at $t=1.756 \mathrm{~ms}$ and $M_{s}=1.1, u_{q, 0}=58.21 \mathrm{~m} / \mathrm{s}, \rho_{p}=664.4$ $\mathrm{kg} / \mathrm{m}^{3}$; (a) $d_{p}=10 \mathrm{~nm}$, (b) $d_{p}=1 \mu \mathrm{m}$, (c) $d_{p}=10 \mu \mathrm{m}$, and (d) $d_{p}=50 \mu \mathrm{m}$.
FIG. 8. Droplet volume fraction in the one-way formalism. Numerical results (blue solid line), theoretical model (red solid line), and maximum $\left(\tau_{v, \max } / \tau_{v, 0}\right)$ for different diameters at $t=1.756 \mathrm{~ms}$, $M_{s}=1.1$, and $\rho_{p}=664.4 \mathrm{~kg} / \mathrm{m}^{3} ;$ (a) $d_{p}$ $=10 \mathrm{~nm}$, (b) $d_{p}=1 \mu \mathrm{m}$, (c) $d_{p}=10 \mu \mathrm{m}$, and (d) $d_{p}=50 \mu \mathrm{m}$. 


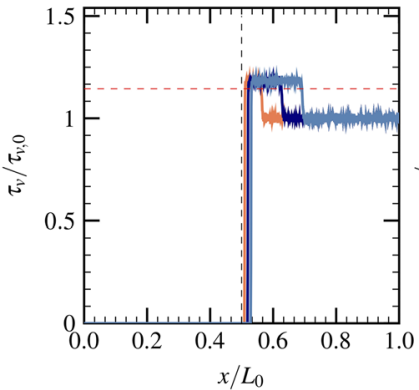

(a)

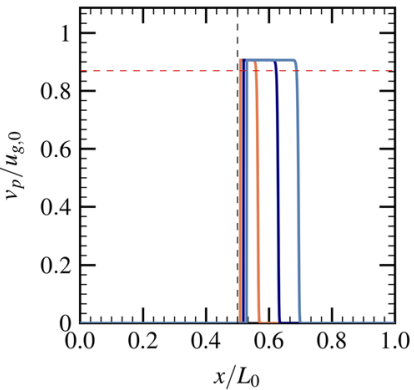

(b)
FIG. 9. Comparison between the theoretical model and numerical simulations at $0.2 \mathrm{~ms}$ (orange solid line), $0.4 \mathrm{~ms}$ (dark blue solid line), $0.6 \mathrm{~ms}$ (blue solid line), theoretical results (red dashed line); $\tau_{v, 0}=5.2 \times 10^{-4}, d_{p}=1 \mu \mathrm{m}, \rho_{p}=664.4 \mathrm{~kg} / \mathrm{m}^{3}$, original contact surface (black dashed line); (a) droplet volume fraction and (b) droplet velocity evolution.

The time necessary for this relaxation process is $\tau_{p}$ [see Figs. 7 (c) and $7(\mathrm{~d})$ ], which increases with their diameters. A comparison between analytical and numerical results shows a good agreement in terms of gas and particle velocities (see Fig. 7).

Figure 8 shows comparisons of the temporal evolutions of the cloud density $\tau_{v}$ between the numerical simulations and the analytical model given by Eq. (21) for particle cloud of different diameters. Different from the continuous solution given by the analytical model, the numerical results show some oscillatory behavior as a result of the random repartition of particles in the Lagrangian formalism used in the Navier-Stokes code. The mean cloud density is seen to be close to the analytical prediction, which is limited by the maximal cloud density obtained by Eq. (21).

The small particles respond immediately to the gas flow [see Figs. 8(a) and 8(b)], while the larger ones accelerate progressively [see Figs. 8(c) and 8(d)]. It can be concluded that the relationship established before in a one-way formalism is validated by the numerical simulations.

The extended two-way theoretical model is studied by comparison with the numerical simulations using two-way formalism as given in Fig. 9. The volume fraction evolution of the particles is shown in Fig. 9(a) for particles of diameter $1 \mu \mathrm{m}$. The maximal value for the volume fraction increases from $5.2 \times 10^{-4}$ to $6.08 \times 10^{-4}$. Similarly, Fig. 9(b) shows the comparison of particle velocities, which increases sharply toward a maximal value that is lower than the initial post-shock gas velocity. The theoretical particle velocity is slightly smaller than the calculation, which results in a lower estimation of the volume fraction as shown in Fig. 9(a). In fact, Eq. (24) can only give a global estimation of the real particle velocity. The relative error of the volume fraction is $2 \%$ in the case of $1 \mu \mathrm{m}$.

\section{NUMERICAL RESULTS}

\section{A. One-way vs two-way simulations}

In this section, the comparison of numerical results using oneway and two-way formalisms is given. Figure 10(a) shows the evolution of volume fraction of particles in the computational domain

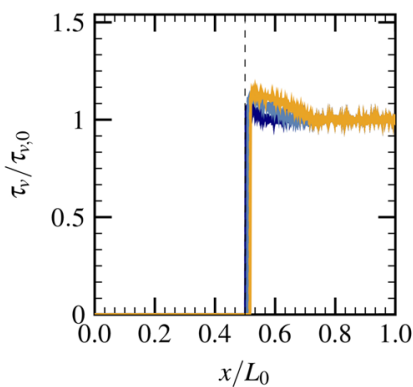

(a)

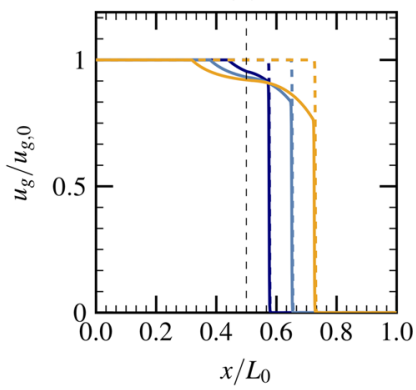

(c)

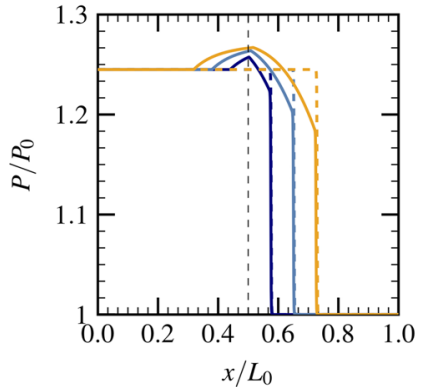

(b)

(d)

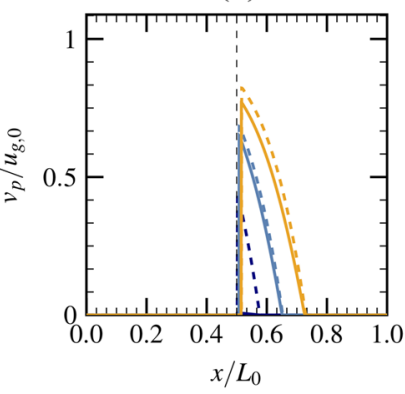

FIG. 10. Comparison one-way/two-way for different time instants. One-way on $0.2 \mathrm{~ms}$ (dark blue dashed line), $0.4 \mathrm{~ms}$ (blue dashed line), and $0.6 \mathrm{~ms}$ (orange dashed line) and two-way on $0.2 \mathrm{~ms}$ (dark blue solid line), on $0.4 \mathrm{~ms}$ (blue solid line), $0.6 \mathrm{~ms}$ (orange solid line); $\tau_{v, 0}=5.2 \times 10^{-4}, d_{p}=10 \mu \mathrm{m}, \rho_{p}=10^{3} \mathrm{~kg} / \mathrm{m}^{3}$, original contact surface (black dashed line); (a) droplet volume fraction, (b) gas pressure, (c) gas velocity, and (d) droplet velocity.

for $t=200-600 \mu \mathrm{s}$. It can be seen that the volume fraction of the particles increases after the passage of the shock. The amplification of the high volume fraction is around 1.1 times the original volume fraction. The interface of the pure gas and the particle-laden domain is pushed downstream of the gas flow. The mass density of particles takes the value of $\rho_{p}=10^{3} \mathrm{~kg} / \mathrm{m}^{3}$ in the following simulations.

Figure 10(b) shows the pressure evolution in the computational domain. Results of the two-way simulations are highlighted by solid lines, while the corresponding one-way simulations are depicted by dashed lines. First, as a result of the attenuation effects of particles, one can notice that the pressure of the post-shock gas is lower than the one-way coupling. This shows that the strength of the shock is decreased due to the presence of particles. Second, the reflection pressure waves are seen only in the two-way simulation. The maximal value for the post-shock pressure is 1.27 bar located at the interface of the two domains. Moreover, the reflection pressure wave propagates at a velocity lower than the original shock wave.

Figure 10(c) shows the evolution of the gas velocity. The oneway simulation indicates that there is no change in the post-shock gas velocity, while this quantity is much reduced in the two-way method, with a maximal velocity of gas smaller than $55 \mathrm{~m} / \mathrm{s}$. An effective change of particle velocity can be seen in Fig. 10(d) for the two-way simulation. After the passage of the shock, the particle velocities are smaller in the two-way simulation compared to the one-way case. 
The comparison indicates that the two-way formalism should be taken into account to better describe the interaction process between the shock wave and the particle cloud.

\section{B. Effects of particle response time}

It is noted that several characteristics of the cloud such as the characteristic response time $\tau_{p}$ and the volume fraction of particle $\tau_{v, 0}$ can have important effects on the interaction mechanism. These effects are studied numerically in this part.

Figure 11(a) shows the gas velocity evolution after the passage of the shock wave through the cloud. Different particle sizes are simulated to elucidate the effect of the response time. The interaction between the particles and the shock can effectively decelerate the post-shock gas velocity. For example, the velocity is reduced from $55 \mathrm{~m} / \mathrm{s}$ to $50 \mathrm{~m} / \mathrm{s}$ for particles having a diameter of $1 \mu \mathrm{m}$ and a volume fraction of $\tau_{v, 0}=5.2 \times 10^{-4}$. The small particles respond rapidly to the shock wave, and give a piece-wise structure of the gas properties during the shock-particle interaction. The larger particles are more difficult to accelerate; thus, they reduce gradually the gas velocity.

The evolution of the particle volume fraction after the passage of the shock is given in Fig. 11(b). One can see that the small particles can give an upper bound of cloud density for the larger ones, which confirms the statement deduced from Eq. (21) through an analytical model.

\section{Effect of particle volume fraction}

The last section concerns the study of the effect of the particle volume fraction. Figure 12(a) shows the gas velocity evolution for different particle volume fractions. The reduction of the gas velocity is much reinforced by the increase in the particle volume fraction. However, the reflected and the transmitted wave velocities seem to be independent of the volume fraction. For a very dense cloud, where $\tau_{v, 0}=5 \times 10^{-3}$, the post-shock gas velocity reduces to zero at $600 \mu \mathrm{s}$, which means that there is no more transmitted pressure wave.

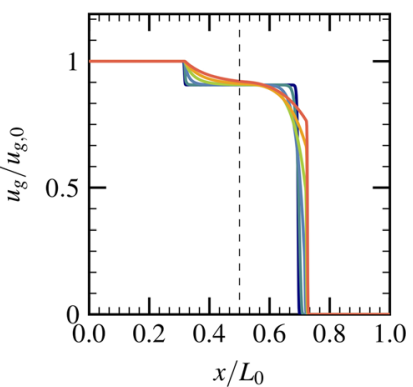

(a)

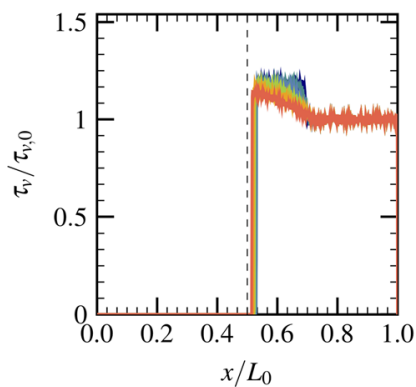

(b)
FIG. 11. Evolutions of flow parameters for different particle diameters: $d_{p}=1 \mu \mathrm{m}$ (dark blue solid line), $d_{p}=2 \mu \mathrm{m}$ (dark green solid line), $d_{p}=4 \mu \mathrm{m}$ (blue solid line), $d_{p}=6 \mu \mathrm{m}$ (green solid line), $d_{p}=8 \mu \mathrm{m}$ (orange solid line), $d_{p}=10 \mu \mathrm{m}$ (red solid line); $t=600 \mu \mathrm{s}, \tau_{v, 0}=5.2 \times 10^{-4}, M_{s}=1.1, \rho_{p}=10^{3} \mathrm{~kg} / \mathrm{m}^{3}$, original contact surface (black dashed line); (a) gas velocity evolution, (b) droplet velocity evolution.

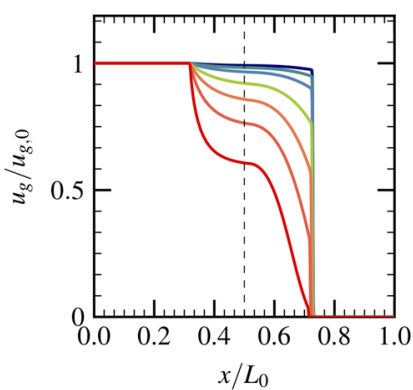

(a)

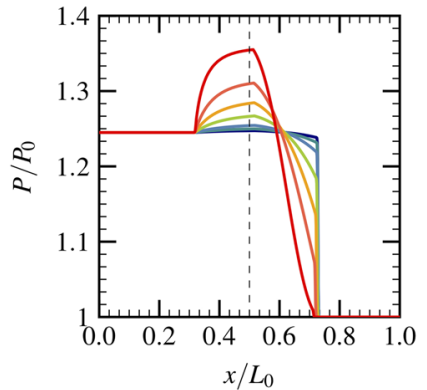

(b)
FIG. 12. Evolutions of flow parameters for different particle volume fractions: $\tau_{v, 0}=5 \times 10^{-5}$ (dark blue solid line), $\tau_{v, 0}=1 \times 10^{-4}$ (dark green solid line), $\tau_{v, 0}=2 \times 10^{-4}$ (blue solid line), $\tau_{v, 0}=5 \times 10^{-4}$ (green solid line), $\tau_{v, 0}=1 \times 10^{-3}$ (orange solid line), $\tau_{v, 0}=2 \times 10^{-3}$ (dark orange solid line), $\tau_{v, 0}=5 \times 10^{-3}$ (red solid line); $t=600 \mu \mathrm{s}, d_{p}=10 \mu \mathrm{m}, M_{s}=1.1, \rho_{p}=10^{3} \mathrm{~kg} / \mathrm{m}^{3}$, original contact surface (black solid line); (a) gas velocity and (b) gas pressure.

Figure 12(b) gives the pressure evolution after the interaction between a shock and the cloud of diameter $d_{p}=10 \mu \mathrm{m}$. One can notice that the particles of volume fraction $\tau_{v, 0}=5 \times 10^{-5}$ have less influence on the pressure evolution. A higher volume fraction $\tau_{v, 0}=5 \times 10^{-3}$ shows an evident pressure increase at the interface between the pure gas and the particle-laden region. It seems that the transmitted pressure is completely attenuated at around $x=0.75 \mathrm{~m}$ in this case. The reflection pressure wave can be noted for all particle volume fractions, and the velocity of the reflected wave seems to be very close.

The comparison shows that the particle volume fraction can enhance the reflection pressure value and play an important role in the attenuation of the transmitted shock.

\section{CONCLUSIONS}

An analytical model is developed to study the cloud topology after the passage of a shock wave in the framework of a one-way interaction formalism. Special attention is made to the momentum exchange between the shock and particles in order to elucidate the dynamic aspects of the shock-cloud interaction mechanisms. The assessment of the model is conducted through a comparison with numerical simulations performed using a high accuracy Navier-Stokes solver.

The extension of the one-way analytical model to the two-way formulation is proposed and compared to the numerical two-way simulations. The two-way theoretical model shows less accuracy than the one-way modeling, but still remains predictable in the scope of this study.

The necessity of using the two-way formalism in the numerical simulation of the shock-cloud interaction is discussed. Various mechanisms such as shock reflection and attenuation can be observed in the two-way simulations, which are neglected in the one-way formalism.

Small particles of diameter $\mathscr{O}(1) \mu \mathrm{m}$ are more sensitive to the drag of the post-shock gas and the present piece-wise structures of 
the shock-cloud interaction. An important shock attenuation effect is noticed for the particle cloud of high volume fractions $\mathscr{O}\left(10^{-3}\right)$.

More studies can be performed considering the two- or threedimensional shock-spray interactions to study the role of the transverse waves on the spray dispersion. The polydispersion of the cloud particles as well as the secondary breakup of the water spray can also be included in the simulations to improve the spray dispersion modeling.

\section{ACKNOWLEDGMENTS}

The authors gratefully acknowledge the financial support from Electricité de France (EDF) within the framework of the Generation II and III reactor research program.

\section{APPENDIX A: CHARACTERISTICS OF THE PLANAR SHOCK}

A planar shock wave can be generated by a piston as shown in Fig. 13. The piston starts moving at $t=0$ with a velocity $V_{p}$, generating a shock wave with a velocity $V_{s}$. Two areas are divided by the shock wave: the post- (1) and the pre-shock area (2). Given the sound speed in the pre-shock area, $c_{2}$, one can obtain the piston velocity, $V_{p}$, by the following relation:

$$
\frac{2}{\gamma+1} \frac{M_{s}^{2}-1}{M_{s}}=\frac{V_{p}}{c_{2}} ; \quad V_{s}=M_{s} c_{2} .
$$

The post-shock gaseous flow is assumed to have the same velocity as that of the piston. Analytical solutions are available for the relationship of the pre- and post-shock thermodynamic quantities, ${ }^{3 .}$

$$
\frac{p_{1}}{p_{0}}=\Gamma_{1}\left(M_{s}, \gamma\right), \frac{T_{1}}{T_{0}}=\frac{\Gamma_{1}\left(M_{s}, \gamma\right) \Gamma_{2}\left(M_{s}, \gamma\right)}{M_{s}^{2}}, \frac{\rho_{1}}{\rho_{0}}=\frac{p_{1}}{p_{0}} \frac{T_{0}}{T_{1}},
$$

where

$$
\Gamma_{1}\left(M_{s}, \gamma\right)=\frac{2}{\gamma+1}\left(\gamma M_{s}^{2}-\frac{\gamma-1}{2}\right), \Gamma_{2}\left(M_{s}, \gamma\right)=\frac{2}{\gamma+1}\left(1+\frac{\gamma-1}{2} M_{s}^{2}\right) \text {. }
$$

\section{APPENDIX B: RESOLUTION OF EQ. (8)}

Equation (8) has the form

$$
t^{\prime}\left(M_{s} c-u_{g}\right)=u_{g} \tau_{p}\left(\exp \left(-\frac{t^{\prime}}{\tau_{p}}\right)-1\right)+M_{s} c t-x
$$

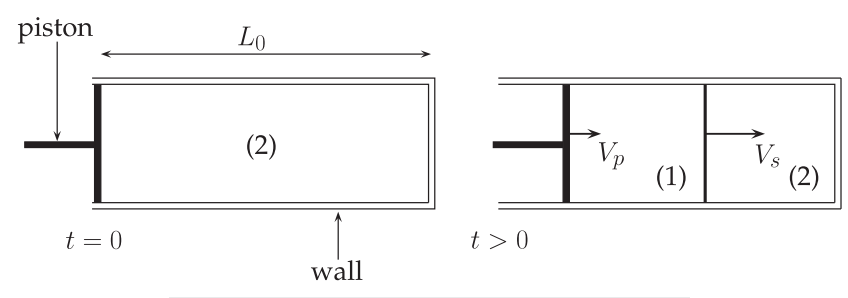

FIG. 13. Shock wave generation in a piston tube. which can be written, by the arrangement of terms, as

$$
\begin{gathered}
{[\underbrace{\frac{t^{\prime}}{\tau_{p}}-\frac{M_{s} c t-x-u_{g} \tau_{p}}{\tau_{p}\left(M_{s} c-u_{g}\right)}}_{\alpha}] \exp (\overbrace{\frac{t^{\prime}}{\tau_{p}}-\frac{M_{s} c t-x-u_{g} \tau_{p}}{\tau_{p}\left(M_{s} c-u_{g}\right)}}^{\alpha})} \\
=\underbrace{\frac{u_{g}}{M_{s} c-u_{g}} \exp \left(-\frac{M_{s} c t-x-u_{g} \tau_{p}}{\tau_{p}\left(M_{s} c-u_{g}\right)}\right)}_{\beta} .
\end{gathered}
$$

The previous equation can also be written as $\alpha \exp (\alpha)=\beta$. We obtain, thanks to the $\mathscr{W}$ Lambert function, ${ }^{30} \alpha=\mathscr{W}(\beta)$. As a consequence, one can obtain

$$
t^{\prime}=\tau_{p} \mathscr{W}\left[\frac{u_{g}}{M_{s} c-u_{g}} \exp \left(-\frac{M_{s} c t-x-u_{g} \tau_{p}}{\tau_{p}\left(M_{s} c-u_{g}\right)}\right)\right]+\frac{M_{s} c t-x-u_{g} \tau_{p}}{M_{s} c-u_{g}} .
$$

\section{REFERENCES}

${ }^{1}$ G. Carrier, "Shock waves in a dusty gas," J. Fluid Mech. 4, 376-382 (1958).

${ }^{2}$ G. Jourdan, L. Biamino, C. Mariani, C. Blanchot, E. Daniel, J. Massoni, L. Houas, R. Tosello, and D. Praguine, "Attenuation of a shock wave passing through a cloud of water droplets," Shock Waves 20, 285-296 (2010).

${ }^{3}$ T. Theofanous, V. Mitkin, and C.-H. Chang, "The dynamics of dense particle clouds subjected to shock waves. Part 1. Experiments and scaling laws," J. Fluid Mech. 792, 658-681 (2016).

${ }^{4}$ J. Kersey, E. Loth, and D. Lankford, "Effect of evaporating droplets on shock waves," AIAA J. 48, 1975-1986 (2010).

${ }^{5}$ O. Williams, T. Nguyen, A.-M. Schreyer, and A. Smits, "Particle response analysis for particle image velocimetry in supersonic flows," Phys. Fluids 27, 076101 (2015).

${ }^{6} \mathrm{G}$. Rudinger, "Some properties of shock relaxation in gas flows carrying small particles," Phys. Fluids 7, 658-663 (1964).

${ }^{7}$ M. Olim, G. Ben-Dor, M. Mond, and O. Igra, "A general attenuation law of moderate planar shock waves propagating into dusty gases with relatively high loading ratios of solid particles," Fluid Dyn. Res. 6, 185-199 (1990).

${ }^{8}$ J. Geng, A. V. de Ven, Q. Yu, F. Zhang, and H. Grönig, "Interaction of a shock wave with a two-phase interface," Shock Waves 3, 193-199 (1994).

${ }^{9}$ Y. Ling, L. Wagner, S. Beresh, S. Kearney, and S. Balachandar, "Interaction of a planar shock wave with a dense particle curtain: Modeling and experiments," Phys. Fluids 24, 113301 (2012).

${ }^{10} \mathrm{~J}$. McFarland, W. Black, J. Dahal, and B. Morgan, "Computational study of the shock driven instability of a multiphase particle-gas system," Phys. Fluids 28, 024105 (2016).

${ }^{11}$ B. Gelfand, "Droplet break-up phenomena in flows with velocity lag," Prog. Energy Combust. Sci. 22, 201-265 (1996).

${ }^{12}$ A. Hadjadj and O. Sadot, "Shock and blast waves mitigation," Shock Waves 23, $1-4$ (2013).

${ }^{13}$ A. Britan, H. Shapiro, M. Liverts, G. Ben-Dor, A. Chinnayya, and A. Hadjadj, "Macro-mechanical modelling of blast wave mitigation in foams. Part I: Review of available experiments and models," Shock Waves 23, 5-23 (2013).

${ }^{14}$ E. D. Prete, A. Chinnayya, L. Domergue, A. Hadjadj, and J.-F. Haas, "Blast wave mitigation by dry aqueous foams," Shock Waves 23, 39-53 (2013).

${ }^{15}$ G. Jourdan, C. Mariani, L. Houas, A. Chinnayya, A. Hadjadj, E. D. Prete, J.-F. Haas, N. Rambert, D. Counilh, and S. Faure, "Analysis of shock-wave propagation in aqueous foams using shock tube experiments," Phys. Fluids 27, 056101 (2015).

${ }^{16}$ A. Foissac, J. Malet, M. Vetrano, J. Buchlin, S. Mimouni, F. Feuillebois et al., "Droplet size and velocity measurements at the outlet of a hollow cone spray nozzle," Atomization Sprays 21, 893-905 (2011). 
${ }^{17} \mathrm{G}$. Thomas, "On the conditions required for explosion mitigation by water sprays," Process Saf. Environ. 78, 339-354 (2000).

${ }^{18}$ G. Gai, S. Kudriakov, A. Hadjadj, E. Studer, and O. Thomine, "Modeling pressure loads during a premixed hydrogen combustion in the presence of water spray," Int. J. Hydrogen Energy 44, 4592-4607 (2019).

${ }^{19}$ T. Hanson, D. Davidson, and R. Hanson, "Shock-induced behavior in micronsized water aerosols," Phys. Fluids 19, 056104 (2007).

${ }^{20}$ A. Chauvin, G. Jourdan, E. Daniel, L. Houas, and R. Tosello, "Experimental investigation of the propagation of a planar shock wave through a two-phase gas-liquid medium," Phys. Fluids 23, 113301 (2011).

${ }^{21} \mathrm{M}$. Pilch and C. Erdman, "Use of breakup time data and velocity history data to predict the maximum size of stable fragments for acceleration-induced breakup of a liquid drop," Int. J. Multiphase Flow 13, 741-757 (1987).

${ }^{22}$ D. Guildenbecher, C. Lopez-Rivera, and P. Sojka, "Secondary atomization," Exp. Fluids 46, 371-402 (2009).

${ }^{23} \mathrm{Y}$. Ling, A. Haselbacher, and S. Balachandar, "Importance of unsteady contributions to force and heating for particles in compressible flows. Part 2: Application to particle dispersal by blast waves," Int. J. Multiphase Flow 37, 1013-1025 (2011).

${ }^{24}$ Y. Mehta, T. Jackson, J. Zhang, and S. Balachandar, "Numerical investigation of shock interaction with one-dimensional transverse array of particles in air," J. Appl. Phys. 119, 104901 (2016).

${ }^{25}$ J. Dahal and J. McFarland, "A numerical method for shock driven multiphase flow with evaporating particles," J. Comput. Phys. 344, 210-233 (2017).
${ }^{26}$ J. Wagner, S. Beresh, S. Kearney, W. Trott, J. Castaneda, B. Pruett, and M. Baer, "A multiphase shock tube for shock wave interactions with dense particle fields," Exp. Fluids 52, 1507-1517 (2012).

${ }^{27}$ Y. Sugiyama, H. Ando, K. Shimura, and A. Matsuo, "Numerical investigation of the interaction between a shock wave and a particle cloud curtain using a CFDDEM model," Shock Waves 29, 499-510 (2018).

${ }^{28} \mathrm{~K}$. Wingerden and B. Wilkins, "The influence of water sprays on gas explosions. Part 2: Mitigation," J. Loss Prev. Process Ind. 8, 61-70 (1995).

${ }^{29}$ S. Elghobashi, "An updated classification map of particle-laden turbulent flows," in IUTAM Symposium on Computational Approaches to Multiphase Flow (Springer, 2006), Vol. 81, pp. 3-10.

${ }^{30}$ R. Corless, G. Gonnet, D. Hare, D. Jeffrey, and D. Knuth, "On the Lambert W function," Adv. Comput. Math. 5, 329-359 (1996).

${ }^{31}$ E. Chang and K. Kailasanath, "Shock wave interactions with particles and liquid fuel droplets," Shock Waves 12, 333-341 (2003).

${ }^{32} \mathrm{O}$. Thomine, "Development of multi-scale methods for the numerical simulation of biphasic reactive flows," Ph.D. thesis, University of Rouen, France, 2011.

${ }^{33}$ G.-S. Jiang and C.-W. Shu, "Efficient implementation of weighted ENO schemes," J. Comput. Phys. 126, 202-228 (1996).

${ }^{34} \mathrm{~A}$. Wray, "Minimal storage time-advancement schemes for spectral methods," Technical Report No. MS 202, NASA Ames Research Center, 1991.

${ }^{35}$ F. White, Fluid Mechanics, McGraw-Hill Series in Mechanical Engineering (McGraw-Hill, 2011). 